Universidad Popular

\title{
El marketing de destino. Percepción del municipio de San Pedro Cholula, Puebla como destino turístico
}

\author{
Laura Mayela Ramírez Murillo , Alicia Lucrecia Yañez Moneda
}

${ }^{1}$ Universidad Popular Autónoma del Estado de Puebla. lauramayela.ramirez@upaep.mx

\section{Resumen}

El destino turístico para sobresalir en este mundo competitivo tiene que tener un sello especial que lo haga único a los productos sustitutos que existen en el mercado. Este trabajo muestra a través de la teoría del marketing de destinos que se deriva del marketing relacional y utilizando una investigación empírica el caso del municipio de San Pedro Cholula, ubicado en el estado de Puebla en el centro de la República Mexicana, como la utilización de la mercadotecnia de relaciones puede aportar aspectos importantes al turismo. Los resultados obtenidos permiten descubrir que es la necesidad de conocimiento sobre el producto turístico el elemento más importante para que ésta localidad haya incrementado en los últimos años a sus visitantes, especialmente porque los consumidores actualmente se interesan por la historia del lugar, sus festividades y tradiciones.

Palabras clave: Marketing de destinos, destino turístico, municipios, distribuidores de productos turísticos.

\section{Target marketing. Perception of the municipality of San Pedro Cholula, Puebla as a tourist destination}

For a tourist destination to excel in this competitive world, it must have a special characteristic that makes it unique from its substitute products. This paper shows, using both the Theory of Destination Marketing, which is derived from Relationship Marketing, and an empiric research about the case of the Municipality of San Pedro Cholula, located in the State of Puebla at the center of the Mexican Republic, how the use of Relationship Marketing is able to contribute important aspects to Tourism. The obtained results allow us to realize that the necessity of knowledge about the tourist product is the most important element so that this location had incremented its number of visitors during the last few years, especially because consumers interest in the history, festivities, and traditions.

Keywords: Destination Marketing, touristic destination, municipalities, tourist products distributors. 


\section{INTRODUCCIÓN}

La vinculación entre el marketing de destinos y el turismo se ha visto enriquecido en los últimos años debido al incremento de personas ansiosas y dispuestas a vivir nuevas experiencias. Los cambios en las tecnologías de la información, el acceso indiscriminado de la información de nuevas rutas y lugares turísticos, el incremento del tiempo libre y la prolongación de la vida de las personas son algunos de los factores que han generado esta mayor vinculación.

El marketing de destino es una evolución del marketing de relaciones y este a su vez es la evolución del marketing de servicios. Según el Kotler y Armstrong (2011) la Mercadotecnia de relaciones surge como resultado de un proceso de construcción de lazos entre los involucrado en una transacción. En pocas palabras son un conjunto de interacciones en donde se desarrollan un encuentro de confianza y compromiso entre las partes (Sánchez y col. 2000; Bordonaba y Redonde, 2006; Kúster y col. 2008; Ferrell y Hartline, 2012).

El marketing de destino al ser una extensión del marketing de relaciones permite establecer relaciones duraderas con todos los públicos involucrados en la oferta del destino o producto turístico como son las relaciones con los proveedores, las relaciones internas de las propias empresas involucradas con sus empleados; las relaciones laterales de los ofertantes como los competidores, organizaciones no lucrativas y gobierno y por último el cliente mismo (Bigné y col. 2000; Pons y col. 2000; Córdova, 2009; San Martín y Matos, 2011).

La presente investigación tiene como objetivo analiza el marketing de destino del municipio del San Pedro Cholula para determinar la efectividad de los proveedores de servicios y la de los distribuidores del mismo que permitan al cliente tener una percepción positiva tanto de los activos tangibles como de los activos intangibles de dicho destino. No se estudiarán por esta ocasión las relaciones de los clientes internos de los ofertantes (empleados) y las relaciones laterales con el gobierno y las organizaciones no lucrativas, estas serán estudiadas en futuras investigaciones. Se seleccionó al municipio de San Pedro Cholula por ser una de las zonas turísticas de mayor demanda del estado de Puebla.

Las aportaciones de esta investigación servirán a las ofertantes del destino turístico a analizar la percepción de los consumidores sobre la existencia y calidad de los servicios en esta región del país.

\section{REVISIÓN DE LA LITERATURA}

Marketing de relaciones \& marketing de destinos

Bordonaba y Redondo (2006) consideran al marketing de relaciones como una de las áreas más desarrolladas y vigentes en la actualidad, ya que permite proporcionarle a la organización un valor agregado. Además, autores como Sánchez y col. (2000) y Ferrell y Hartline (2012) hablan de que la mercadotecnia de relaciones es una filosofía para establecer relaciones fructíferas con agentes del mercado, utilizando elementos clave como la personalización y la comunicación para generar compromiso y confianza.

Sánchez y col. (2000), explica que los factores que propiciaron el desarrollo del marketing de relaciones fueron: en primer lugar, la intensificación de la competencia; en segundo lugar, la necesidad de fidelizar a los clientes; la concepción de los mercados como redes; desarrollo tecnológico y, el agotamiento del marketing de servicios, en donde el servicio fue sustituido por la relación. Por lo que Ferrel y Hartline (2012) han resumido lo más importante del marketing de relaciones en siete principios: centrado en retener a los clientes; orientado hacia los beneficios del producto; plantea una visión a largo plazo, gran énfasis en el servicio al cliente; alto nivel de compromiso, alto nivel de contactos con el cliente y; la calidad es una preocupación de todos.

En el caso de Martín (2004) el marketing de relaciones es un proceso continuo en donde no se concibe la venta del bien o servicio como el final de la relación, sino que además se realizan una serie de actuaciones en la postventa para agradar, revitalizar, reactivar y fidelizar a los clientes. Para Córdoba (2009) existen diez relaciones de intercambio básicas de las empresas. La empresa-cliente; la empresadistribuidores; la empresa-empleados; la empresadepartamentos; la empresa-unidades de negocios; la empresa-proveedores; la empresa-proveedores de servicios; la empresa-competidores; la empresa-organizaciones no lucrativas y; la empresa-gobierno.

El marketing de destinos es una extensión del marketing de relaciones, en el cual se establecen relaciones duraderas con todos los involucrados en la planeación, desarrollo y promoción de un destino turístico y no como incorrectamente se ha definido como la promoción turística o la publicidad o ferias turísticas. En este sentido Bigné y col. 2000 establece que el marketing de destinos es la satisfacción de las necesidades de los turistas y la comunidad local, en donde el sector público y privado ha aplicado no solo parte del marketing mix tradicional al turismo sino a la generación de relaciones a largo plazo con todos los públicos del destino turístico. 
En este sentido el marketing de destino son todas las comunicaciones, experiencias, activos tangibles (infraestructura) e intangibles (emociones y percepciones) y las relaciones que se establecen entre ellos para generar una oferta turística particular que genere un destino turístico o producto turístico (Pons y col. 2000). San Martín y Matos (2011) consideran que un destino turístico basa su posicionamiento en los beneficios emocionales que el cliente recibe, tales como felicidad y alegría que a la larga generará en un vínculo emocional del cliente con el destino turístico. Además consideran que los determinantes de la intención de ir a un destino turístico son la confianza y el compromiso de todos los involucrados en la oferta de los mismos.

\section{METODOLOGÍA}

En esta investigación se ha optado por analizar la percepción de los consumidores de destinos turísticos. Se decidió elegir la relación de los mismos con los proveedores de servicios y los distribuidores. No se consideran ni las relaciones internas (empleados) y las relaciones laterales (ONGs y gobierno).

La investigación es empírica se aplicó al destino turístico Municipio de San Pedro Cholula (región centro de la República Mexicana). Para la investigación se realizaron 100 encuestas de los turistas de la comunidad. Para analizar la percepción del destino turístico desde el punto de vista del marketing relacional y el marketing de destinos se utilizó en modelo de Morgan y Hunt (1994) citado en Córdova (2009), (Tabla 1).

Tabla 1. Modelo de marketing de destinos

\begin{tabular}{|c|c|c|}
\hline DIMENSIÓN & CLAVE & CARACTERÍSTICAS \\
\hline \multirow{5}{*}{$\begin{array}{l}\text { Conocimientos } \\
\text { del destino turístico }\end{array}$} & CD1 & Conoce usted los diferentes atractivos del municipio de San Pedro Cholula? \\
\hline & CD2 & ¿Ha visitado anteriormente el Municipio de San Pedro Cholula? \\
\hline & CD3 & Visita este Municipio por algunas Festividades? \\
\hline & CD4 & Su mayor gusto por visitarlo es por conocer su historia? \\
\hline & CD5 & La promoción de este destino en revistas especializadas, lo invitó a conocerlo. \\
\hline \multirow{10}{*}{$\begin{array}{l}\text { Relación de los } \\
\text { proveedores }\end{array}$} & RP1 & ¿Por medio de amigos o familiares conoció este destino? \\
\hline & RP2 & Conoció la promoción de este destino en revistas especializadas. \\
\hline & RP3 & ¿Cree que la actual presidencia municipal promueva de forma correcta a San Pedro Cholula como destino? \\
\hline & RP4 & ¿Existen productos turísticos que complementan el desarrollo de su visita en este municipio? \\
\hline & RP5 & Según su percepción, ¿existe un equilibrio entre la oferta de los prestadores de servicios y los servicios existentes? \\
\hline & RP6 & ¿Presentan los medios informativos toda la gama de productos del Municipio? \\
\hline & RP7 & Conoce usted el municipio por medio de alguna agencia de viajes instalada en un local \\
\hline & RP8 & Usted busca en medios digitales para localizar los productos turísticos que ofrece el municipio. \\
\hline & RP9 & $\begin{array}{l}\text { Cuál de los siguientes medios publicitarios tradicionales utiliza para visitar los atractivos turísticos. Televisión } \\
\text { 3) Módulos de turismo 4) periódico 5) Recomendación boca a boca }\end{array}$ \\
\hline & RP10 & 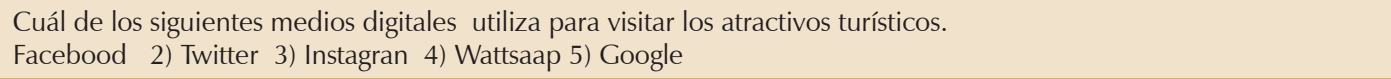 \\
\hline \multirow{10}{*}{$\begin{array}{l}\text { Relación con } \\
\text { distribuidores }\end{array}$} & RD1 & ¿Existen suficientes hoteles que satisfagan de manera adecuada la demanda del turista? \\
\hline & RD2 & ¿La imagen del destino es exactamente como fue describió en su difusión? \\
\hline & RD3 & ¿Considera que el Municipio de San Pedro está posicionado a nivel Nacional? \\
\hline & RD4 & ¿Existen elementos innovadores para que usted siga viniendo al Municipio de San Pedro? \\
\hline & RD5 & ¿Ha recibido usted una atención personalizada en algunos servicios turísticos. \\
\hline & RD6 & ¿La señalética y caminos están perfectamente identificados para llegar al destino sin ningún contratiempo? \\
\hline & RD7 & ¿Existen servicios de internet en el sitio donde se hospedó? \\
\hline & RD8 & ¿Los lugares que visitó en el Municipio de San Pedro son fácilmente localizables por Google Maps? \\
\hline & RD9 & ¿Los restaurantes que visitó en el Municipio le permiten acceder a una reservación por un código QR? \\
\hline & RD10 & ¿Conoce usted alguna publicidad de este Municipio que se filmara con drones, videos u otra tecnología? \\
\hline
\end{tabular}

Fuente: Elaboración propia. Adaptado de Morgan y Hunt (1994) citado en Córdoba (2009). Del marketing transaccional a marketing relacional.

Entramado, 9: 6-17. 
La ficha técnica del trabajo de campo aparece en la tabla 2.

Tabla 2. Técnica del estudio y características de la muestra

\begin{tabular}{|c|c|}
\hline Población & $\begin{array}{l}\text { Turistas que acuden al municipio de San Pedro } \\
\text { Cholula, Puebla. Nacionalidad mexicana el } 75 \% \text {. } \\
\text { Nacionalidades extranjeras } 25 \% \text {. }\end{array}$ \\
\hline Área geográfica & $\begin{array}{l}\text { Centro de la República Mexicana. Municipio } \\
\text { conurbano a la Ciudad de Puebla. }\end{array}$ \\
\hline Tamaño muestral & $\begin{array}{l}\text { Se recibieron } 100 \text { encuetas válidas de } 112 \\
\text { encuestas con un margen de error }+/-5 \% \text {. }\end{array}$ \\
\hline Procedimiento & Muestreo aleatorio simple \\
\hline $\begin{array}{l}\text { Características } \\
\text { de la muestra }\end{array}$ & $\begin{array}{l}\text { Sexo femenino el } 63 \% \text {; masculino } 37 \% \\
\text { Edad: Entre los } 20 \text { a los } 65 \text { años. } \\
\text { Nivel de estudios: } 35 \% \text { posee estudios medios, } \\
\text { el } 65 \% \text { estudios de licenciatura y el } 5 \% \text { estudios } \\
\text { de posgrado. } \\
\text { Profesión: el } 22 \% \text { trabajadores por cuenta } \\
\text { propia; el } 38 \% \text { empleados; el } 27 \% \text { estudiantes } \\
\text { y } 23 \% \text { jubilados. }\end{array}$ \\
\hline $\begin{array}{l}\text { Fecha de trabajo } \\
\text { de campo }\end{array}$ & Del 20 de agosto al 20 de octubre del 2019 \\
\hline $\begin{array}{l}\text { Administración } \\
\text { de la encuesta }\end{array}$ & Personal, con cuestionario estructurado \\
\hline
\end{tabular}

Fuente: Adaptado de Landero, R; González, M. (2006) Estadística con SPSS y Metodología de la Investigación. México. Editorial Trillas

\section{HIPÓTESIS PLANTEADAS}

Ha1. Las personas visitan San Pedro Cholula porque desean conocer más sobre la historia y tradiciones de este destino turístico.

Ha2. Los proveedores de servicios de San Pedro Cholula han permitido que este destino turístico cuente con la calidad necesaria para darle confianza al consumidor.

Ha3. Los distribuidores de servicios de San Pedro Cholula han permitido que este destino turístico cuente con la calidad necesaria para darle confianza al consumidor.

\section{RESULTADOS EMPÍRICOS}

Con el fin de analizar las hipótesis propuestas se ha desarrollado un análisis estadístico en tres etapas: en la primera, se analiza la validez y confiabilidad del instrumento utilizado; en la segunda se realizó el análisis de tabla de contingencia, y; en la tercera, se realizó el análisis descriptivo usual y el análisis factorial de la muestra de la población objetivo. El programa empleado fue el SPSS versión 24.

La siguiente tabla muestra el análisis de confiabilidad en donde se puede apreciar que el Alfa de Cronbach de cada una de las dimensiones y mayor al 0.65 necesario para que el instrumento sea válido (Tabla 3).
Tabla 3. Confiabilidad del instrumento de recolección de datos.

\begin{tabular}{ll} 
CATEGORÍAS & ALFA DE CRONBACH \\
\hline Conocimiento del destino turístico & .461 \\
\hline Proveedores & .697 \\
\hline Distribuidores & .829 \\
\hline Total & .885 \\
\hline
\end{tabular}

La confiabilidad del instrumento es válida debido a que todos los valores del Alfa de Cronbach son mayores a 0.65 .

Fuente: Elaboración propia.

La siguiente tabla muestra el análisis de validez del instrumento en donde se establece que si las correlaciones de las dimensiones son menores al Alfa de Cronbach se puede considerar que el instrumento es válido (Tabla 4).

Tabla 4. Validación del instrumento de recolección de datos

$\begin{array}{|lccc|} & \text { Conocimientos } & \text { Proveedores } & \text { Distribuidores } \\ \text { Conocimientos } & .461 & & \\ \text { Proveedores } & .662 & .697 & \\ \text { Distribuidores } & .706 & .644 & .829\end{array}$

** La correlación es significativa al nivel 0,01 (bilateral). La correlación entre conocimiento del destino y proveedores es de .662 menor al Alfa de Cronbach de .697; las correlaciones entre conocimiento y distribuidores es de .706; y la correlación los proveedores y los distribuidores es de .644 son menores al Alpha de Cronbach que es de .829. Fuente: Elaboración propia.

\section{ANÁLISIS DE TABLAS DE CONTINGENCIA}

La tabulación cruzada elaborada fue la siguiente: Tabla de contingencia entre el sexo y el ingreso de los turistas del municipios de San Pedro Cholula; tabla de contingencia entre el tipo de viaje que realizan los turistas y su edad, y; entre el número de años viajes al año y la edad de los turistas.

En la tabla 5 de se puede apreciar la tabla de contingencia entre el ingreso y el sexo de los encuestados. Las mujeres con ingresos entre 16,000 a 21,000 pesos son las que más predominaron en el destino turístico, mientras que los hombres con un ingreso mayor a los 22,000 pesos son los que más se encuestaron en este destino turístico. Adicionalmente el valor de la Chi-cuadrada es de 1.639, por lo que se determina que las variables no son independientes, lo que implica que el ser hombre o mujer si afecta el ingreso que ellos obtienen. 
Tabla 5. Tabla de contingencia entre el sexo e ingreso.

\begin{tabular}{lcc} 
Ingreso & Mujeres \% & Hombres \% \\
\hline Menos de 10,000 & $47.8 \%$ & $52.2 \%$ \\
\hline Entre 11,000 a 15,000 & $46.7 \%$ & $53.3 \%$ \\
\hline Entre 16,000 a 21,000 & $60 \%$ & $40 \%$ \\
\hline Más 22,000 & $37.5 \%$ & $62.5 \%$
\end{tabular}

Las mujeres con ingresos entre 16,000a 21,000 son las que realizan viajes a este municipio; mientras que los hombres con ingresos mayores a 22,000 realizan más este tipo de viajes.

En la tabla 6 de se puede observar la tabla de contingencia entre el tipo de viaje que realizan los turistas y su edad. Las personas con edades entre 20 y 30 años y de 31 a 40 años realizan el viaje por motivos familiares en un $50 \%$; las personas mayores a 41 años realizan los viajes por motivos de recreación. Adicionalmente el valor de la Chi-cuadrada es de 6.743, por lo que se determina que las variables no son independientes, lo que implica que el tipo de viaje depende de la edad de los turistas.

Tabla 6. Tabla de contingencia entre el tipo de viaje y edad.

\begin{tabular}{|lccc|}
\hline Tipo de viaje & Trabajo & Recreación & Familiar \\
\hline De 20 a 30 años & $13.6 \%$ & $36.4 \%$ & $50 \%$ \\
\hline De 31 a 40 años & $21.4 \%$ & $28.6 \%$ & $50 \%$ \\
\hline De 41 a 50 años & $18.2 \%$ & $63.6 \%$ & $18.2 \%$ \\
\hline 50 en adelante & $16.7 \%$ & $66.7 \%$ & $16.7 \%$ \\
\hline
\end{tabular}

Los turísticas más jóvenes son los que realizan sus viajes por motivos familiares, mientras los turistas con mayor edad realizan sus viajes por recreación.

En la tabla 7 de se puede observar la tabla de contingencia entre el ingreso y el número de viajes que los turistas realizan al año. Las personas con ingresos menores a 10,000 pesos realizan de 3 a 5 viajes al año; las personas con ingresos entre 11,000 a 15,000 realizan viajes de 1 a 2 veces año. Las personas con ingresos entre 16,000 a 21,000 de 3 a 5 veces al año. Y por último, las personas con ingresos mayores a 22,000 realizan más de 9 viajes al año. Adicionalmente el valor de la Chi-cuadrada es de 43.39, por lo que se determina que las variables no son independientes, lo que implica que el ingreso determina cuántas veces al año viajan los turistas.

Tabla 7. Tabla de contingencia entre ingreso y número de viajes al año.

\begin{tabular}{lcccc}
$\begin{array}{l}\text { Ingreso \& número de } \\
\text { viajes año }\end{array}$ & $\begin{array}{c}\text { De 1 a 2 } \\
\text { viajes }\end{array}$ & $\begin{array}{c}\text { De } 3 \text { a } 5 \\
\text { viajes }\end{array}$ & $\begin{array}{c}\text { De } 6 \text { a } 8 \\
\text { viajes }\end{array}$ & $\begin{array}{c}\text { Más } \\
\text { de } 9\end{array}$ \\
\hline Menos de 10,000 & $32.6 \%$ & $52.2 \%$ & $8.7 \%$ & $6.5 \%$ \\
\hline Entre 11,000 a 15,000 & $40 \%$ & $26.7 \%$ & $26.7 \%$ & $6.7 \%$ \\
\hline Entre 16,000 a 21,000 & $50 \%$ & $50 \%$ & $0 \%$ & $0 \%$ \\
\hline Más 22,000 & $12.5 \%$ & $0 \%$ & $0 \%$ & $87.5 \%$
\end{tabular}

Las personas con menores ingresos viajes de 1 a 2 veces al año; las personas con mayores ingresos viajan más de 9 veces al año.

\section{ANÁLISIS DESCRIPTIVO}

Con respecto a la muestra de los turistas que turistas que acuden al municipio de San Pedro Cholula, Puebla. Con un $75 \%$ de nacionalidad mexicana y un $25 \%$ de nacionalidad extranjera se efectuó el análisis descriptivo habitual y frecuencia del uso de las redes sociales (Tablas $8,9$ y 10$)$.

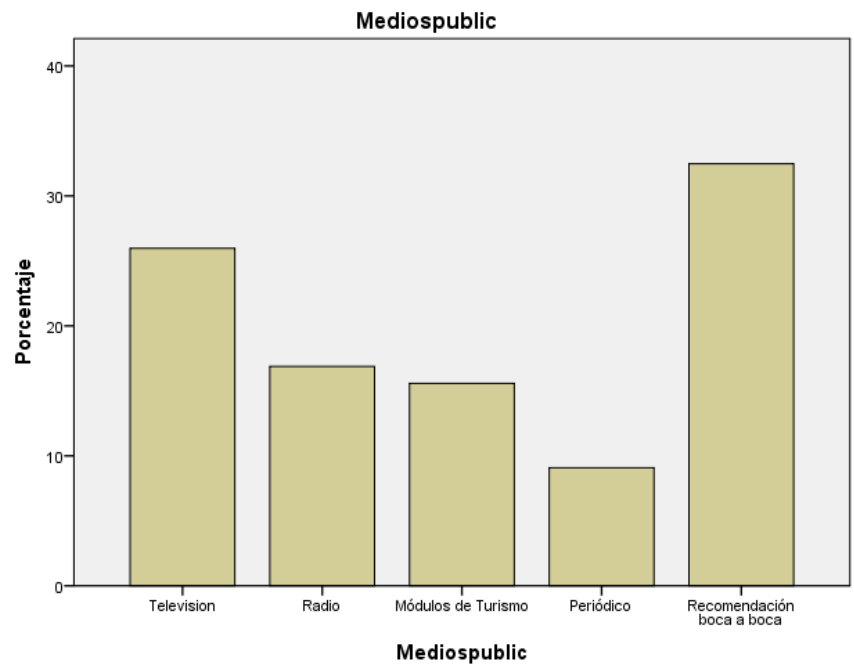

En la tabla 8 se puede observar que los medios convencionales más utilizados más utilizados son: información de boca de boca con un 33 \&, le sigue la televisión con 27 \% y por último el radio con un $18 \%$.

En la tabla 9 se puede analizar que los medios digitales más utilizados son: Facebook con un 45 \%, Google con $23 \%$ y Twitter con un $16 \%$.

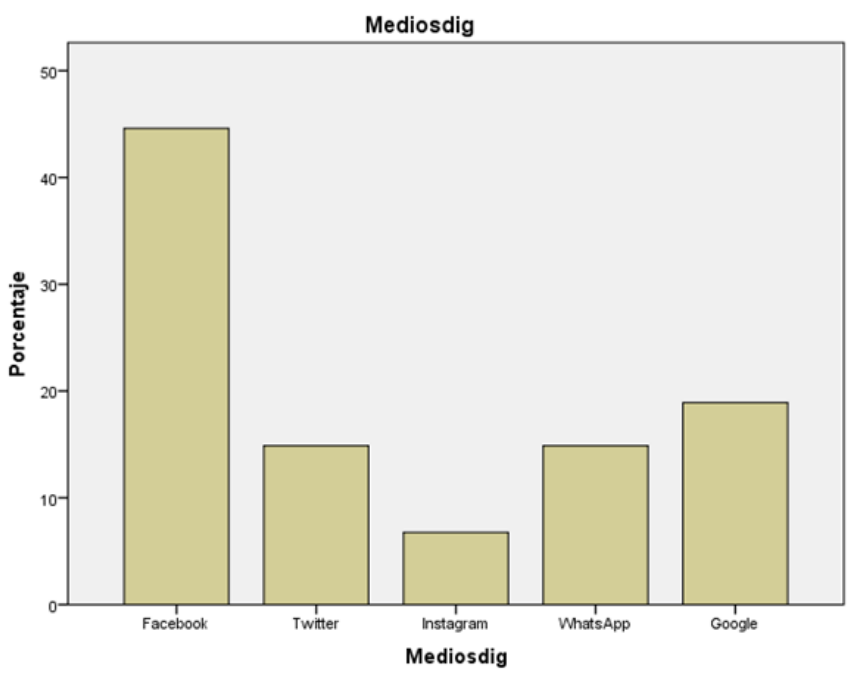

Por lo que se refiere al análisis estadístico se pudo comprobar que la media más importante es la de la dimensión 1 con 
3.46. Considerando esta dimensión como la de mayor impacto en considerar a San Pedro Cholula como un destino turístico adecuado. Mientras que la de menor impacto es la percepción que se tiene de los proveedores (Tabla 10).

Tabla 10. Estadísticos descriptivos, media y desviación estándar

\begin{tabular}{|c|c|c|c|c|}
\hline Dimensión & Clave & $\mu$ & $\sigma^{2}$ & Promedio \\
\hline \multirow{5}{*}{ Conocimiento } & CD1 & 3.32 & 1.39 & \multirow{5}{*}{3.46} \\
\hline & CD2 & 3.79 & 1.32 & \\
\hline & CD3 & 3.48 & 1.49 & \\
\hline & CD4 & 3.39 & 1.48 & \\
\hline & CD5 & 3.33 & 1.59 & \\
\hline \multirow{10}{*}{ Proveedores } & RP1 & 3.19 & 1.56 & \multirow{10}{*}{3.24} \\
\hline & RP2 & 3.30 & 1.53 & \\
\hline & RP3 & 3.71 & 1.33 & \\
\hline & RP4 & 3.47 & 1.36 & \\
\hline & RP5 & 3.43 & 1.42 & \\
\hline & RP6 & 3.06 & 1.59 & \\
\hline & RP7 & 3.65 & 1.44 & \\
\hline & RP8 & 3.07 & 1.62 & \\
\hline & RP9 & 3.05 & 1.62 & \\
\hline & RP10 & 2.49 & 1.66 & \\
\hline \multirow{10}{*}{ Distribuidores } & RD1 & 3.00 & 1.55 & \multirow{10}{*}{3.45} \\
\hline & RD2 & 3.60 & 1.03 & \\
\hline & RD3 & 3.64 & 1,29 & \\
\hline & RD4 & 3.31 & 1.45 & \\
\hline & RD5 & 3.57 & 1.44 & \\
\hline & RD6 & 3.60 & 1.31 & \\
\hline & RD7 & 3.23 & 1.55 & \\
\hline & RD8 & 3.87 & 1.24 & \\
\hline & RD9 & 3.31 & 1.43 & \\
\hline & RD10 & 3.38 & 1.49 & \\
\hline
\end{tabular}

Fuente: Adaptado de Landero, R; González, M. (2006) Estadística con SPSS y Metodología de la Investigación. México. Editorial Trillas

Adicionalmente see utilizó la técnica de interdependencia conocida como factorial (Morales, 2013). Esta técnica permite determinar los factores de mayor impacto, es decir, cuáles son las dimensiones que perciben los turistas de San Pedro Cholula como destino. Se utilizó el método de componentes principales, en el cual se observa que la varianza total de los datos es del $66.6 \%$ y que seis factores explican las variables que impactan al destino. De acuerdo a la medida de Kaiser-Meyer-Olkin (KMO) puede decirse que el modelo factorial utilizado es apropiado, ya que el índice $\mathrm{KMO}$ es de $0.720(0.720>=.50)$ indicando que se trata de un buen modelo factorial, ya que las variables no se correlacionan y que un factor no se encuentra incluido en otro. En la Tabla 11 se observa que el factor más importante que afectan al destino turístico San Pedro Cholula. De acuerdo a este análisis se pueden detectar cuatro factores, el primero llamado atracción del municipio engloba las variables de atracción como el saber sobre las tradiciones y fiestas, que los servicios son de calidad y que se puede utilizar el código QR en varios establecimiento. El factor 2 llamado medios, se engloban los principales medios que han ayudado a que este municipio tenga una imagen adecuada y un posicionamiento. El factor 3 llamado festividades, reconoce que las personas van a este municipio por sus festividades a través de lo que observan en revistas especializadas y por medio de las agencias de viaje. Y por último el factor 4 llamado servicios hace referencia a la excelencia del municipio en presentar sus festividades y la calidad de sus hoteles (Tabla 11).

Tabla 11. Matriz de componentes rotados

\begin{tabular}{|c|c|c|c|c|}
\hline Variables & $\begin{array}{l}\text { Factor } 1 . \\
\text { Atracción }\end{array}$ & $\begin{array}{l}\text { Factor } 2 . \\
\text { Medios }\end{array}$ & $\begin{array}{c}\text { Factor3. } \\
\text { Festividades }\end{array}$ & $\begin{array}{l}\text { Factor } 4 . \\
\text { Servicios }\end{array}$ \\
\hline Conocer atractivos & 0.530 & & & \\
\hline $\begin{array}{l}\text { Equilibrio entre } \\
\text { prestadores del } \\
\text { servicio y calidad } \\
\text { del servicio }\end{array}$ & 0.685 & & & \\
\hline Código QR & 0.632 & & & \\
\hline $\begin{array}{l}\text { Adecuados } \\
\text { medios de } \\
\text { promoción }\end{array}$ & & 0.673 & & \\
\hline $\begin{array}{l}\text { Adecuados } \\
\text { medios digitales }\end{array}$ & & 0.615 & & \\
\hline $\begin{array}{l}\text { Imagen del } \\
\text { destino exacta }\end{array}$ & & 0.643 & & \\
\hline $\begin{array}{l}\text { Posicionamiento } \\
\text { adecuado }\end{array}$ & & 0.509 & & \\
\hline $\begin{array}{l}\text { Atención } \\
\text { personalizada }\end{array}$ & & & 0.500 & \\
\hline $\begin{array}{l}\text { Conoce } \\
\text { festividades }\end{array}$ & & & 0.565 & \\
\hline $\begin{array}{l}\text { Revistas } \\
\text { especializadas } \\
\text { promueven }\end{array}$ & & & 0.670 & \\
\hline Agencias de viajes & & & 0.567 & \\
\hline $\begin{array}{l}\text { Excelentes medio } \\
\text { tradicionales }\end{array}$ & & & & 0.734 \\
\hline Hoteles & & & & 0.655 \\
\hline
\end{tabular}

Aquí se presentan los cuatro factores resultantes del modelo factorial. El primer factor se denomina atracción laboral en donde destacan aspectos como la necesidad de los turistas de conocer las tradiciones y costumbres de los lugares que visitan; el segundo factor, llamado medios, en donde se destaca la necesidad que el destino turístico tanga adecuados medios tradicionales y medios digitales; el factor 3 , reconocer a las festividades como una forma de atracción al municipios; el cuarto factor, servicios, implica la necesidad de que los distribuidores de los servicios turísticos sean de excelente calidad. Fuente: Elaboración propia. 


\section{CONCLUSIONES}

El estudio empírico realizado obtuvo los siguientes resultados. Las percepciones de los turistas que consideran al municipio de San Pedro Cholula como destino turístico son positivas. Resalta de manera importante que los turistas en la actualidad no solo quieren conocer los lugares turísticos de una manera superficial, la tendencia en estos momentos es que los visitantes se quieran involucrar en mayor medida con las comunidades, es decir, están deseosos por conocer las festividades, tradiciones e historia de los lugares a los que acuden.

Los resultados apoyan las hipótesis propuestas, sin embargo es la hipótesis 1 es la que tiene mayor impacto en la investigación. Ya que las personas visitan San Pedro Cholula porque desean conocer más sobre la historia y tradiciones de este destino turístico. La hipótesis menos impactante es que los proveedores de servicios de San Pedro Cholula han permitido que este destino turístico cuente con la calidad necesaria para darle confianza al consumidor, esto se debe a que ellos no requieren esmerarse en modificar los productos turísticos existentes ya que es venden por sí mismos y los turistas que visitan el Municipio no exigen productos diferentes dentro de los ya existentes.

Es importante recalcar que mientras otros destinos exigen el uso de tecnologías para identificar los sitios de interés, en San Pedro Cholula no se requiere de ellos porque hasta la fecha ha influido más la recomendación del sitio de boca en boca y televisión. Sería de gran utilidad que las autoridades del Municipio consideraran generar spots publicitarios en televisión y cine para promocionar su destino. Asimismo es importante recalcar que los turistas actuales quieren vivir experiencias memorables en sitios de interés, por lo que se recomienda el iniciar con campañas del uso de medios digitales como instagram o twitter para que el usuario suban a las redes experiencias del destino.

\section{REFERENCIAS}

Bordonabe, J. V. y Redondo, P. Y. (2006). Marketing de relaciones en los canales de distribución: un análisis empírico. Cuadernos de Economía y Dirección de la Empresa, 29: 5-30.

Bigné, E., Font, X. y André, L. (2000). Marketing de destinos turísticos: análisis y estrategias de desarrollo. Madrid: ESIC.

Córdova, J. (2009). Del marketing transaccional al marketing relacional. Entramado, 9: 6-17.

Ferrell, O. C. y Hartline, M. D. (2012). Estrategias de Marketing. México: Cengage Learning Editores.

Kotler, P., Armstrong, G. P. (2011). Fundamentos del Marketing. México: Pearson Educación.

Kúster, I., Vila, N. y Canales, P. (2008). El marketing relacional y el marketing emocional: Dos enfoques competitivos para el turismo de sol y playa. Estudios sobre el Consumo, 84: 31-40.

Landero, R; González, M. (2006). Estadística con SPSS y Metodología de la Investigación. México: Editorial Trillas

Martín, I. (2004). Dirección y gestión de empresas del sector turístico. Madrid: Pirámide

Morgan, M. y Hunt, D. (1994). The commitment-trust theory of relationship marketing. Journal of Marketing, 15: 22.

Pons, R., Morales, L. y Díaz, Y. (2000). La imagen del destino y comportamiento de compra turística. Retos turísticos, 2: $1-7$.

Sánchez, P. M., Gil, S. I. y Mollá, D. A. (2000). Estatus del Marketing Relacional. Revista Europea de Dirección y Economía de la Empresa, 9: 47-64.

San Martín, S. y Matos, R. (2011). Determinantes de la intención del consumidor de ir a un destino turístico. Aplicación al caso de Mundo, Maya-México. Cuadernos de gestión, 11: 75-93. 